\title{
Integrated ICT System for Teaching Physical Sciences in a Robotic Laboratory
}

\author{
doi:10.3991/ijet.v4s3.1118 \\ M. Tsavli, J. Fragakis, S. Kopsidas, D. Zisiadis and D. Vavougios \\ University of Thessaly, Volos, Greece
}

\begin{abstract}
The Information and Communication Technologies provide economically feasible and effective means to assist individuals with kinetic disabilities in numerous activities concerning educational purposes. As the technology is increasingly used in everyday environments, an early response of the existing methods to teach the Physical Sciences to individuals with kinetic disabilities is our innovative system. The work presented in this article is part of the "Smart and Adaptable Information System for Supporting Physics Experiments in a Robotic Laboratory" (SAIS-PEaRL) research project.
\end{abstract}

Index Terms - experiments in Physics, Information and Communication Technologies, kinetic disabilities, robotic laboratory

\section{INTRODUCTION}

\section{A. Individuals with disabilities}

Special needs [1] is a term used to describe individuals who require assistance for disabilities that may be medical, mental or psychological. Nowadays there are over 750 million individuals of this category worldwide. Throughout history, these individuals have encountered many barriers that have mainly been aroused by the society attitudes. Poor or no education, lack of employment, complete disregard for their needs by the community and stereotypes such as harmful characterizations were prominent in the society. All the students, without any discrimination, ought to have the same education and training opportunities. As far as individuals with kinetic disabilities are concerned, technology helps to overcome some barriers in education, as it offers alternative ways of communication and interaction with the environment.

\section{B. The Information and Communication Technologies}

The Information and Communication Technologies (ICTs) [2] have signaled major improvements in education, employment and everyday life. Undoubtedly they are an exceptional and inextricable tool of modern educational systems worldwide. The main aim of the ICTs in education is to familiarize students with the use of technological equipment such as computers, communication mechanisms, controlling devices, etc. Via the modern educational technologies each student with kinetic disabilities can easily approach knowledge.

The pedagogic role of ICTs can be defined for all students in five levels:

- Training the students in the ICTs as a discrete educational module,
- Using the ICTs as a means to collect information of any form, as a means to communicate with individuals or environments that formerly had been impossible,

- Exploiting the ICTs as an interactive and supervisor means for the goals of the tuition,

- Using the ICTs as a method to broaden knowledge,

- Using the ICTs for entertainment and relaxation.

\section{The Physical Sciences}

Physics is a natural science that studies the matter and its motion through space-time and all that derives from these, such as energy and force. More broadly, it is the general analysis of nature, conducted in order to understand how the world and universe behave.

Physics is a fundamental science due to the fact that many other sciences (biology, geology, chemistry, medicine etc) are based on the laws and concepts of physics. Physical laws and concepts can advance new technologies that make the every day life easier, such as electromagnetism (television, computer, domestic appliances), but can also provide technical means for any concern (motorized transport, heavy industry's mechanisms, calculus, research in astronomy, etc).

The key to completely comprehend the physical laws and concepts is the experiment. Real world phenomena can be investigated by real world experiments as the most effective way to investigate the relations among some variables or test a hypothesis is to perform an experiment. Such experiments are considered fundamental for today's way of life and thinking, as Galileo Galilei's experiment: He used rolling balls to disprove the Aristotelian theory of motion (1602 - 1607), Guglielmo Marconi demonstrated that radio signals can travel between two points separated by an obstacle (1895), the Big Bang Experiment (Large Hadron Collider) which was intended to recreate the conditions a few moments after the Big Bang (2008) [3].

\section{The experiment as a teaching method}

Undoubtedly in order to stimulate the students' interest in the learning subject, the teacher ought to draw the students' attention and excite their will for knowledge. The most effective and interesting educational method for this purpose is the experiment. The experiment underlies a valuable and powerful method to develop and evaluate teaching and learning sequences. The students approach knowledge by setting the experiment components, performing the experiment and observing the reliable results. Many schools have equipped laboratories where students can participate in the experiment performance, even in cases of individuals with kinetic disabilities. 


\section{E. Experiments for students with kinetic disabilities}

The first and most important phase of the experimental procedure is its preparation. Afterwards, the student must initialize the experiment and observe the results. Finally, the experimental area must be cleared in order for the students to prepare for the next experiment. However, in order to have reliable results for observation, the students must setup all the necessary components correctly and with precision. In cases of individuals with kinetic disabilities this process proves to be difficult to achieve, as time and accuracy play a determinant role in the experiment results. The time is limited during a learning session and different settings of the components may lead to different and unreliable results.

A valuable solution can be provided by an ICT system that can identify and set all the components in place, perform the experiment and provide statistical data of the results if requested.

\section{F. Assistive technologies for individuals with kinetic disabilities}

The term Assistive Technology (AT) [4] includes any device, mechanism or complete system which is used to increase, preserve or improve certain functionalities of individuals with disabilities. AT promotes independence by enabling people with disabilities to perform tasks that they were formerly unable to accomplish, or had great difficulty in accomplishing them. Many assistive technology products have been designed in order to assist individuals with kinetic disabilities such as:

- Robotic mechanisms (robotic arms)

- Special interfaces (joysticks, touch pads)

- Accessible computer inputs (ergonomic keyboards, keyboards with big keys, foot operated mouse, trackballs, touch screens)

- Assistive software with input devices (keyboard shortcuts, sticky keys, software for customization of pointer appearance)

\section{RELATED PROJECTS AND RESEARCH}

In this session we present some robotic systems that have been designed for experiment performance execution and introduce interesting techniques. At first there is Adam [5], the robot scientist: a computer system that automates the hypothetico- deductive method and the recording of experiments in sufficient detail to enable reproducibility. Adam's hypotheses have been proved correct and novel by the researchers who used separate manual experiments to test and confirm them.

Another system that performs experiments for educational purposes is the ARALEV project (Autonomous Robotic Arm with Laser Enhanced Vision) [6]: this project uses a robotic arm, vision, and a logical language which is based on three principles: "FIND", "TRACK" and "FETCH", implemented in Java.

Internet-Based Robotics and Mechatronics Experiments for Remote Laboratory Development [7] describes a series of laboratory experiments in internet-based robotics and mechatronics plus the design, development and evaluation of an internet-based laboratory facility. This project uses robots, webcams, sensors, a data acquisition system and software applications. The experiment execution can be performed remotely through the World Wide Web.
Similar to the above mentioned system is the robotic system called the robot turtle Robotnačka [8]. It provides a platform for the students for various experiments and it is supported by collateral software packages, in order to serve the educational needs of every student. Additionally it includes a set of student exercises along with the matching explanations.

An innovative platform that uses a low cost robotic vehicle was designed for both research and education purposes, considering minimum cost [9]. The vehicle, Rogue Blue, suitably modified and accompanied by the proper software was used to perform most of the common research applications and educational tasks.

As far as the learning subject is Physics a laboratory work has been developed in order to achieve this knowledge using a teaching robot arm for mechanics and electronic circuits [10]. Through experiment performance, this system combines the mechanical arrangement with an electronic control circuit and it models an instructional tool in the physics laboratory to teach the law of mechanics and basic electronics for both teacher and students.

Apparently none of the above systems focuses on teaching the Physics concepts and laws. Moreover these systems are not accessible to individuals with kinetic disabilities. The systems in their majority are difficult to be used by individuals with kinetic disabilities and require time and effort on behalf of both teachers and students.

\section{SYSTEM ARCHITECTURE AND OPERATION}

There were many challenges in order to design a complete robotic system that would teach Physics to individuals with kinetic disabilities. The ICT system that we designed is estimated to overcome any minor problem that may occur. The first phase of the project is the calibration of the controlling devices that compose the system. During the second phase, the student operates the robotic arm in order to perform an experiment. A High-Level ROBOT Language is introduced to assist the students with experiment description and performance. The third phase concerns the possibility of performing an experiment remotely via the World Wide Web. This aspect of the implemented system concerns not only students with kinetic disabilities but also every student who needs access to a robotic laboratory in order to perform experiments from a distance. The last phase involves all the necessary equipment used throughout the experiment performance in order to observe in detail the step-by-step process and the recording of its results.

\section{A. Automating the preparation of robotic arm controlling}

Prior to experiment performance and whenever a student intends to use some special interface in order to operate a robotic arm, the interface/robotic arm calibration must be settled. A different individual with kinetic disabilities needs a new calibration due to the differences in the type of the kinetic disabilities that may have. Whenever many students need to use the robotic arm, many calibrations must be done and this leads to major time loss since calibration can be extremely time consuming.

Such a problem can be dealt with by establishing a database that will store all the necessary data, along with the controlling devices adjustments for each user that were produced during the calibration phase and organizes this 
information in profiles. As a result, the user will not have to calibrate the controlling device every time he/she needs to operate it, but can easily retrieve all the necessary adjustments from the profiles database and the calibration phase can be skipped.

The main components of the system:

1. The Robot Control Station: responsible to collect every user calibration data and stores that data in a database, creating the users' profiles. If the user is already registered the RCS retrieves all the necessary data in order to complete the user's calibration. Through some very simple forms the RCS manages the database providing some functionalities such as update, delete, create. It is the middleware that receives movement signals from the special interfaces and translates them to robotic commands in order to properly control it. It also maintains static data for further analysis.

2. Robotic arm: There is a wide variety of robotic arms in the market. Robotic arms have features that allow movements with precision and can be easily controlled by a user via a great range of controlling devices. They are flexible; they can be adjusted so they are considered as an exceptional tool for experiment performance even in cases of individuals with kinetic disabilities.

3. The Profiles Database: profiles may contain both the calibration data and additional information about the users' preferences. That information may have some educational value since we will be able to extract valuable conclusions. For example, we may conclude that a specific type of interfaces is more appropriate for a specific user with a specific type of kinetics disabilities or that the interface is easier to support users without any experience with the system but after a short term of educational process regarding the use of the system a different interface is more efficient.

4. Special Interfaces: these are the controlling devices, touch pads, joysticks, etc. For example for a user that can move his arm a more appropriate solution is a joystick, or for a user that can move his fingers a better solution is a touch pad.

5. Assistive Proper Software: Software designed according to the needs of the students and the requirements of the system.

6. Ethernet Switch: The connections of the system are established over an Ethernet Local Area Network in order to achieve sufficient data transfers and lowtime (almost real-time) responses. Fig 1.

The system architecture is designed as depicted in

\section{B. Automating the preparation for experiment performance}

An innovative ICT system that automates the process of preparation has been designed in order to prevent from time loss and lack of accuracy in the experiment performance. The main components of the system are the following:

1. The robotic arm

2. The items' RFIDs database

3. The Scripts database

4. Accompanied Proper Software running on the RCS
5. Components and system functionality for calibration

In order to depict the functionality of the system (Fig. 2) we should firstly describe the laboratory's domain and its features:

1. The robotic arm is placed on a rectangular desk and cannot be moved.

2. The desk has a repository which contains objects necessary for the experiment performance and a mapped area which is where the experiments take place.

3. All objects have RFIDs on them.

4. The robotic arm has a RFID reader that can identify the items in a few centimeters range.

5. The objects that are used in the experiments are small and light-weighted.

At first, the tutor counseled by the appropriate software describes the experiment. The description, the source code and the title of the experiment is saved in the experiment scripts database and can be easily recovered when needed. The RFIDs database contains all the RFIDs of the items along with a short description. The robotic arm is connected to the Robotic Control System through a robot control interface.

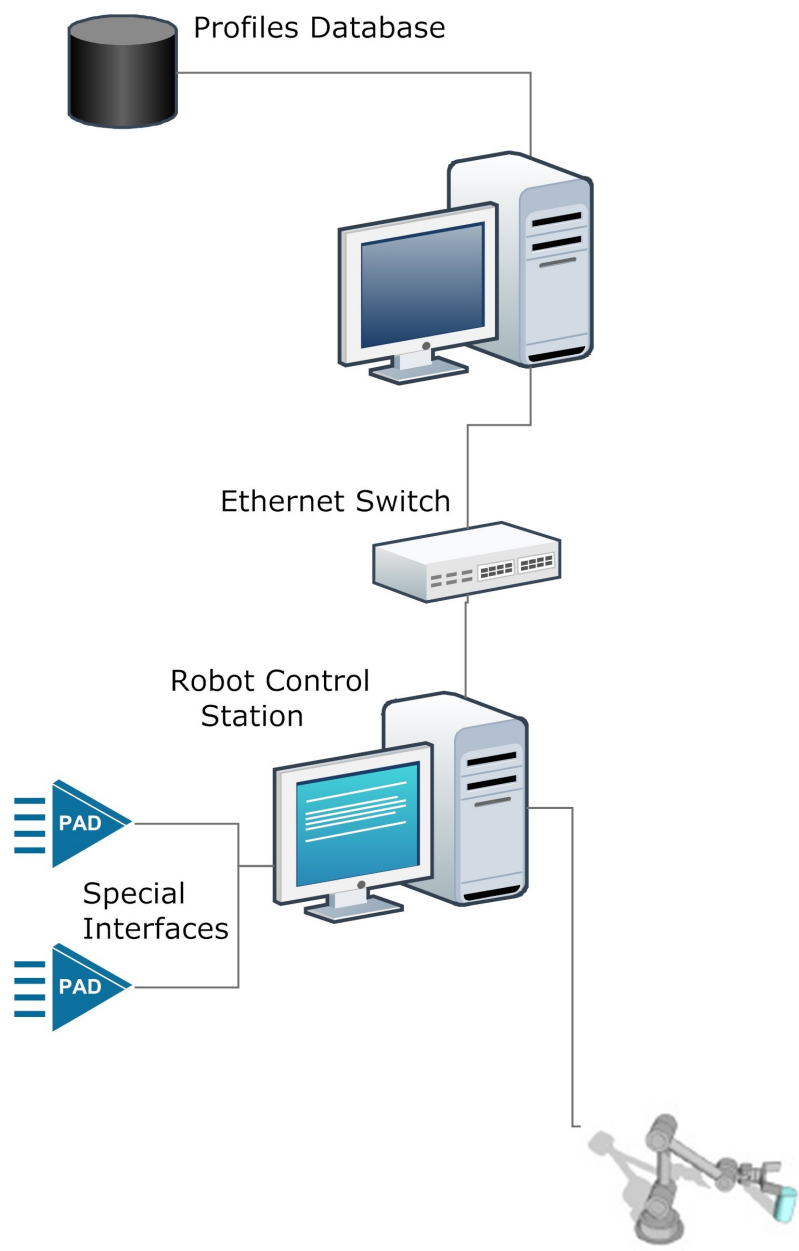

Robotic Arm 


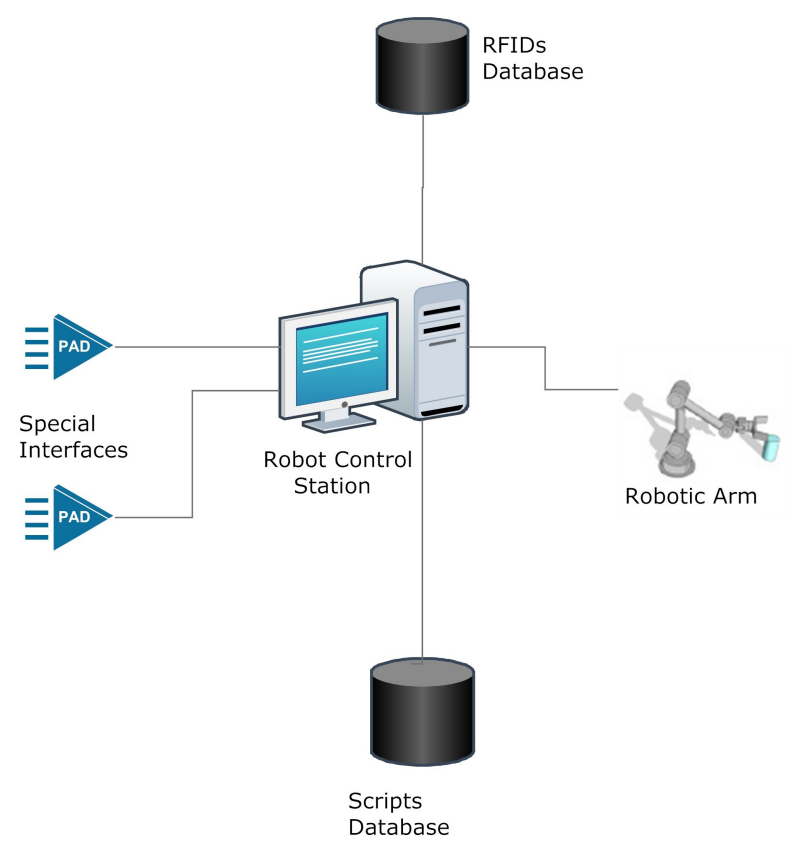

Figure 2. Architecture for the experiment preparation

The Robot Control Station reads a script - this could be a new script or a stored one that has been retrieved from the Experiment Scripts Database - and instructs the robotic arm to place the items that are needed for the experiment in the right positions. When the preparation is completed the student decides whether to perform the experiment himself/herself (manual use of the robotic arm) or to automatically begin the performance by the Robot Control Station.

Apart from the controlling devices, a robot can be programmed to perform a specific task or even a series of tasks. Due to the complexity of the modern robotic languages a new high level programming language has been designed which makes it easy to describe Physics experiments for robots in a laboratory. Each student is enabled to operate the basic robotic arm functionalities according to the degrees of freedom of the specific robotic arm.

The instruction set that constitutes the logical language is described in detail:

DEFINE (area) Prior to starting an experiment, the whole laboratory domain needs to be scanned in order to find where the location of the specific area that is used as an argument in the command is. For example, with the following command:

DEFINE(repository): The arm scans the whole laboratory domain in order to find the whereabouts of the repository, where all the objects are placed at first.

FIND(item): This instruction locates the item used as argument and returns the $\mathrm{x}, \mathrm{y}$ position as a result. This means that the robotic arm spreads and scans the domain in order to be in a close distance from the object to identify it.

PLACE (item, $z, w)$ : This instruction makes the arm scan the repository, find the specified item, move towards it, lower to grab it, raise and move it to the specified position (the next two arguments of the parameters), lower, release it and raise again. More specific this instruction runs the following block of instructions:

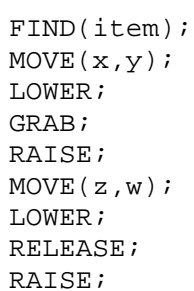

REMOVE(item): In case an object needs to be removed from the experiment domain this instruction should be used. The implementation of this instruction is similar to the PLACE() command, only the last two parameters are no longer needed, as the remove command places the objects in the repository by default.

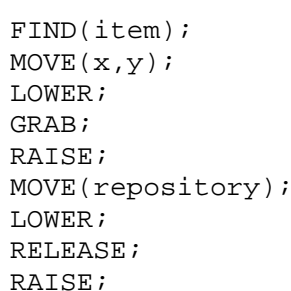

The statements in the for-loop repeat continuously for a specific number of times. Expression_1 is the starting point, expression_2 is the condition that must be true in order to repeat the loop, and expression_3 is the step.

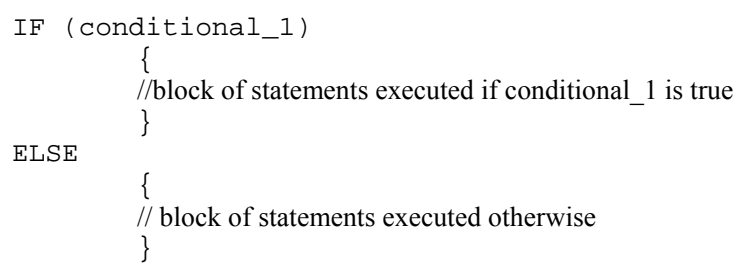

The IF-ELSE statements provide conditional branching for easy and effective control checks. Conditional_1 is the condition that must be true in order to execute the $\bar{b}$ lock of statements into the IF sentence. In any other case the ELSE block of statements is executed.

CLEAR ALL: This instruction is used if the experiment execution has been completed and the experimental domain must empty in order to prepare another experiment. It removes all the objects and places them into the repository. The implementation is based on the REMOVE() command.

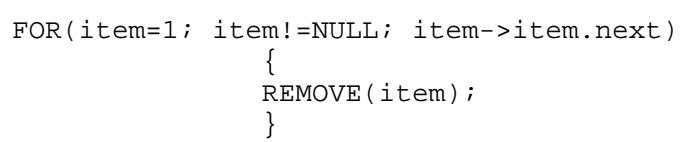

The flexibility of the system lies on the ability to decide whether to retrieve an existing scenario from the scripts database or create and program a new script from the beginning.

\section{Embedding Remote Control Functionalities}

Each student ought to have access to the robotic laboratory in order to perform a physical experiment and observe the results. The described above robotic laboratory is equipped with embedded cameras to depict live the dif- 
ferent aspects of the laboratory area. The student connects to the Virtual LAN (VLAN) of the laboratory platform via the World Wide Web (Figure 3), logins to the platform, loads his/hers profile from the database, inputs the necessary data (instructions) or retrieves an experiment scenario from the scripts database and initializes the experiment. The student observes the real-time experiment performance through the cameras.

In case the student initializes a new experiment he/she is able to store the script in his/her favorite scripts database along with the experimental results from further analysis. The remote control functionality includes access to the scripts database, ability to store new scripts and ability to watch recorded experiments and results executed by other students.

The core elements of the system software are:

1. The database client and server

2. The ROBOT control interface and server side application

3. The script language interface and compiler

4. The video client and server

The main advantage is that the above mentioned robotic system does not offer virtual performance but rather a real-time process, with real conditions and reliable results. Students are enabled to perform experiments just via an internet connection from the comfort of their private space.

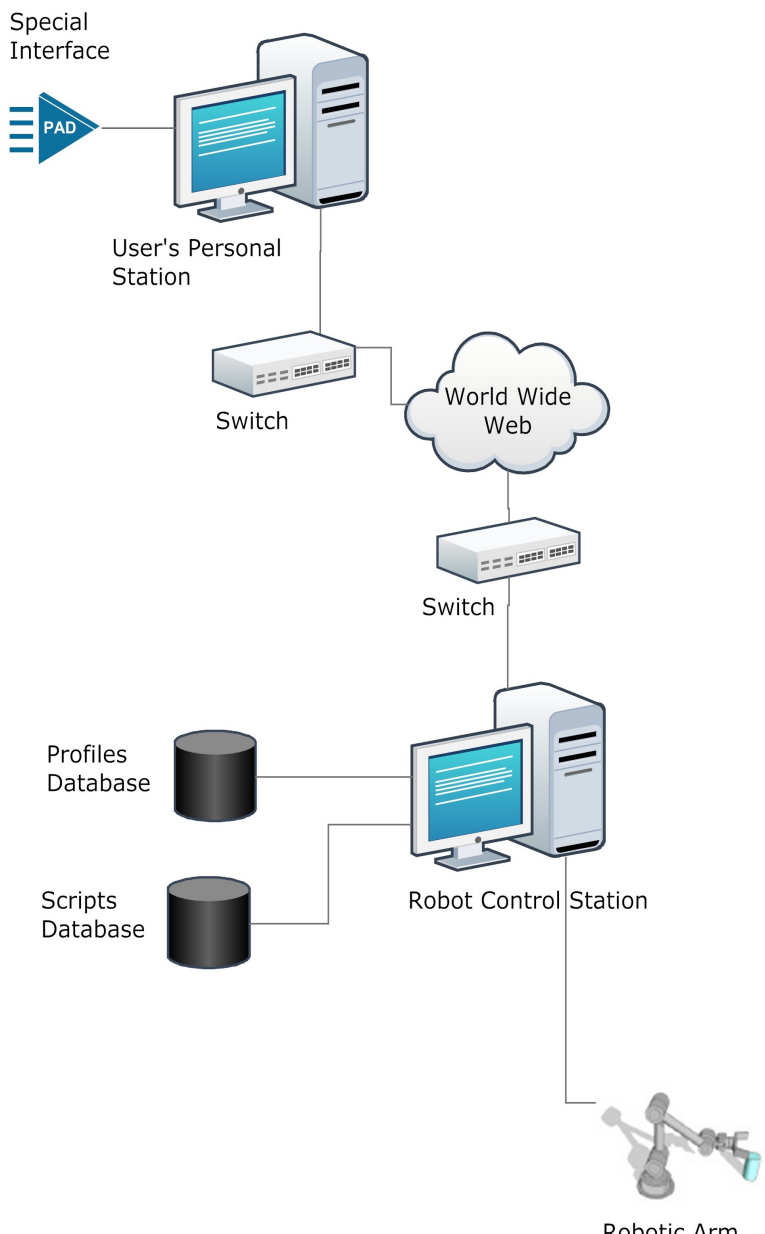

Figure 3. Remote control access to the robotic laboratory

\section{Embedded camera system for data recording}

The robotic laboratory provides further functionalities of data recording for further analysis and evaluation of the experimental results. Special equipment is installed in the laboratory area to provide precise data recording such as thermal infrared imaging cameras or special featured cameras that take a large number of snapshots per second, so in depth analysis is guaranteed.

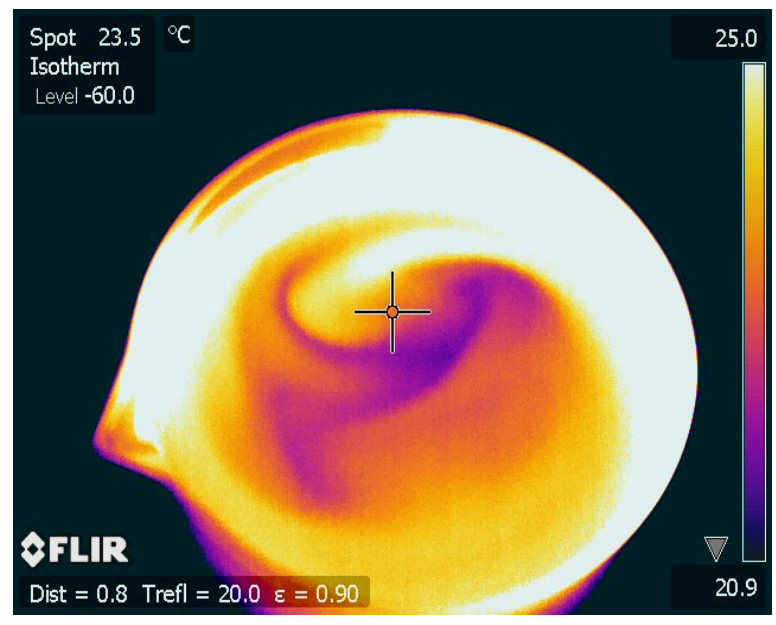

Figure 4. Thermal infrared camera image [18]

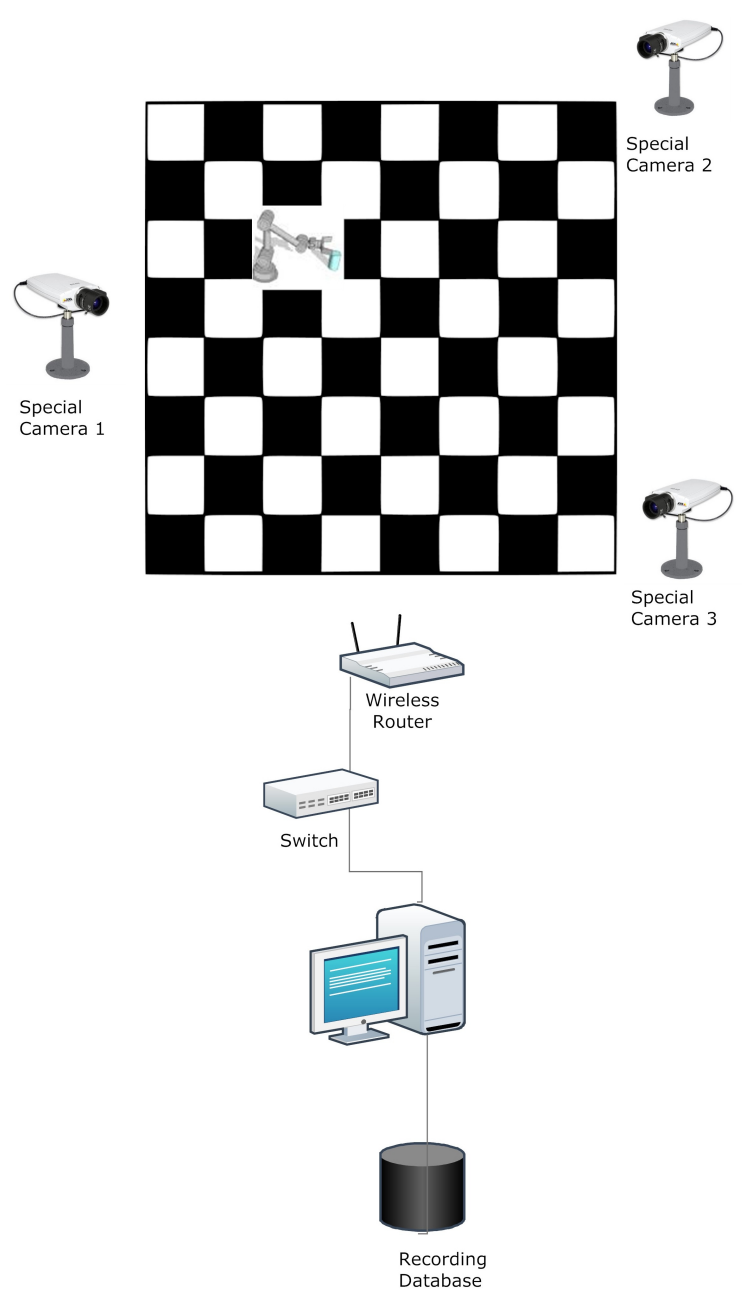

Figure 5. Mapped laboratory area equipped with special cameras 
The following example could provide a better understanding of the determinative and valuable role these recording cameras play. In physics, mechanical energy [11] describes the sum of potential energy and kinetic energy present in the components of a mechanical system and Heat [12] is the process of energy transfer from one body or system due to thermal contact (collision). These types of energy are difficult to grasp with the naked eye of the inexperienced students. A thermal camera can depict the heat energy that appears in the objects right after the collision.

Another example is that of the horizontal throw. The motion is too quick and the naked eye cannot trace the track of the object thrown in detail. A camera that shoots a large number of snapshots per second can be used to record the movement of the object for a more detailed analysis.

\section{Future SUGGESTIONS FOR FURTHER RESEARCH}

As far as future research is concerned, the system offers easy expansion and complete independence of any other software or hardware.

One of the aspects that would be challenging to develop in the future is the enhancement of the types of experiments, not only in the mechanics field but also in other topics of the Physical Sciences, such as electromagnetism or electrical circuits.

A second robotic mechanism (or a second robotic arm) interfering with the robotic laboratory could prove a valuable solution for more complex experiments.

More degrees of freedom in the existing robotic arm would provide more flexibility to the movements of the robotic arm and experiments that require total precision could be performed. Nevertheless, this option would mean the need for further progress in the robotic language and for improvements on several other software features of the ICT system.

A software expansion is under consideration for development that would stimulate the experiment process, record the results and provide statistics of the experiments.

Due to the effectiveness and the flexibility for easy expansion of the system further research will be developed gradually in the near future.

\section{CONCLUSIONS}

In the present work we analyse the architectural fundamentals for the development of an ICT system, which would be able to support the preparation of Physics experiments in a robotic physics laboratory. The proposed system ensures performance accuracy and a high degree of results precision. In addition, it provides an effective, interesting and pleasant way of teaching the Physical laws and concepts to students with kinetic disabilities. Some of the many advantages are:

- Time limited calibration of the robotic arm and preparation of the experiment,

- More students may have enough time to perform an experiment more than once, even in classes with a large number of students,

- In-depth analysis of the experimental results via the special featured cameras,
- Remote control of the robotic arm for students that are away from the robotic laboratory.

\section{REFERENCES}

[1] http://en.wikipedia.org/wiki/Special_needs

[2] http://en.wikipedia.org/wiki/Information_and_communication tec hnologies

[3] http://en.wikipedia.org/wiki/Physics

[4] http://en.wikipedia.org/wiki/Assistive technology

[5] Ross D. King, Jem Rowland, Stephen G. Oliver, Michael Young, Wayne Aubrey, Emma Byrne, Maria Liakata, Magdalena Markham, Pinar Pir, Larisa N. Soldatova, Andrew Sparkes, Kenneth E. Whelan, Amanda Clare King RD, Rowland J, Oliver SG, Young M, Aubrey W, Byrne E, Liakata M, Markham M, Pir P, Soldatova LN, Sparkes A, Whelan KE, Clare A, The Automation of Science, AAAS Science Magazine, Vol. 324. no. 5923, pp. $85-89$

[6] http://rullstol.sm.luth.se/aralev

[7] Richard Chiou, Yongjin Kwon, Shreepud Rauniar, and Horacio Sosa, "Internet-Based Robotics and Mechatronics Experiments for Remote Laboratory Development", ASEE Annual Conference \& Exposition, June 24-27, 2007 - Honolulu, Hawaii

[8] Petrovič, P., Balogh, R., Ličny, A. \& Weiss, R. (2007), “Using Robotnačka in Research and Education", In Eurologo 2007 Conference. FMFI UK.

[9] S. Piperidis, L. Doitsidis, C. Anastasopoulos, and N. C. Tsourveloudis, "A Low Cost Modular Robot Vehicle Design for Research and Education", in CD-ROM proceedings of the IEEE $15^{\text {th }}$ Mediterranean Conference on Control \& Automation, Athens, 2007.

[10] O. Sise, "A laboratory Work: A Teaching Robot Arm for Mechanics and Electronic Circuits", The 4th International Conference on Physics Teaching in Engineering Education PTEE 2005, June 29July 1, 2005, Brno, Czech Republic.

[11] http://en.wikipedia.org/wiki/Mechanical energy

[12] http://en.wikipedia.org/wiki/Heat

[13] http://sais-pearl.sed.uth.gr/

[14] M. Tsavli, S. Kopsidas, J. Fragakis, D. Zisiadis, D. Vavougios, C. Karagiannidis and L. Tassiulas, "Automating the Preparation and the Execution of Physics Experiments in a Robotic Laboratory", International Conference on Education and New Learning Technologies (EDULEARN09), Barcelona, Spain, July 2009.

[15] J. Fragakis, S. Kopsidas, M. Tsavli, D. Zisiadis, D. Vavougios, C. Karagiannidis and L. Tassiulas, "Teaching Physics to Individuals with Kinetic Disabilities Using Robotics: A-State-of-the-Art", International Conference on Education and New Learning Technologies (EDULEARN09), Barcelona, Spain, July 2009.

[16] M. Tsavli, S. Kopsidas, J. Fragakis, D. Zisiadis, C. Karagiannidis, D. Vavougios and L. Tassiulas, "Embedding Remote Control Functionalities into a Robotic Physics Laboratory", International Conference on ICT in Teaching and Learning (ICT2009), Hong Kong, 6-8 July 2009.

[17] ", J. Fragakis, S. Kopsidas, M. Tsavli, D. Zisiadis and D. Vavougios, "New Trends in Physics Education: Performing Experiments in a Robotic Laboratory, 6th International Conference "New Horizons in Industry, Business and Education" (NHIBE09), Santorini, Greece, 27-28 August 2009

[18] http://commons.wikimedia.org

\section{AUTHORS}

M. Tsavli was born in Athens, Greece. She holds a Diploma from the Computer Engineering and Telecommunications Department / University of Thessaly and she currently is a Ph.D. student in the Special Education Department of the same University. Her research interest are in the fields of computer supported learning and educational technologies.

J. Fragakis was born in 1966, in Chania, Greece. He holds a Diploma of Electronics and Computer Engineering from the Technical University of Crete and he currently is 
a Ph.D. student in the Special Education Department/ University of Thessaly. He has served as a Computer Science high school teacher and now he serves as a teaching assistant at the Computer Engineering and Telecommunications Department / University of Thessaly. His research interests include educational information systems, robotics and electronics.

S. Kopsidas was born in Lefkada, Greece. He holds a Diploma from the Electronics and Computer Engineering Department / Technical University of Crete and a Ph.D. from the Computer Engineering and Telecommunications Department / University of Thessaly. He is an associate researcher at the Institute of Telematics and Informatics / Centre for Research and Technology - Hellas (ITI/CERTH). His research interests are in the fields of computer and telecommunications security.

D. Zisiadis was born in Kastoria, Greece. He holds a BSc and an MSc from the Computer Science Department,
University of Crete and a Ph.D. from the Computer Engineering and Telecommunications Department, University of Thessaly. He is author of many research papers and articles and he has participated into a large number of research projects. His main areas of interest are computer networks and mobile \& wireless communications.

D. Vavougios was born in 1957, in Athens, Greece. He obtained the BSc in Physics in 1982 and the Ph.D. in Theoretical Physics in 1989, both from the Physics Department / University of Patras, Greece. He is Assistant Professor in the Department of Special Education, University of Thessaly, Greece, where he teaches Physics and Science Education. His research interests are in the fields of Theoretical Physics and Science Education.

This article was modified from a presentation at the International Conference of Interactive Computer Aided Learning ICL2009, September 2009 in Villach, Austria. Submitted 20 October 2009. Published as resubmitted by the authors on 2 November 2009. 